\title{
Empirically Based Guidelines for Selecting Vagus Nerve Stimulation Parameters in Epilepsy and Heart Failure
}

\author{
Eric D. Musselman, ${ }^{1}$ Nicole A. Pelot, ${ }^{1}$ and Warren M. Grill ${ }^{1,2,3,4}$ \\ ${ }^{1}$ Department of Biomedical Engineering, Duke University, Durham, North Carolina 27708 \\ ${ }^{2}$ Department of Electrical and Computer Engineering, Duke University, Durham, North Carolina 27708 \\ ${ }^{3}$ Department of Neurobiology, Duke University, Durham, North Carolina 27708 \\ ${ }^{4}$ Department of Neurosurgery, Duke University, Durham, North Carolina 27708 \\ Correspondence: warren.grill@duke.edu
}

\begin{abstract}
Vagus nerve stimulation (VNS) is a promising therapy to treat patients with epilepsy and heart failure. Outcomes of preclinical studies and clinical trials indicate that the selection of stimulation parameters has a direct impact on therapeutic efficacy and patient tolerability, suggesting that both the efficacy and tolerability of VNS could potentially be improved with a change in stimulation parameters. In this review, the success of translating stimulation parameters for epilepsy and heart failure from preclinical studies in animal models to human use in the clinic is evaluated on the basis of patient outcomes and stimulation-induced side effects. Data suggest that patients receiving VNS for epilepsy may experience improved seizure reduction by increasing the frequency and/or duty cycle of stimulation as well as incorporating closed-loop systems to deliver stimulation closer to seizure onset. Further, data suggest that VNS for heart failure is limited by the inability to activate the nerve fibers mediating therapeutic benefit without co-activation of side effect-inducing fibers. This may explain why pivotal trials of VNS for heart failure failed to meet primary efficacy outcomes despite promising preclinical outcomes in animal models. Improved characterization of the relationship between the stimulation parameter space and recruitment of the underlying fiber populations will likely expand the use of VNS to treat a variety of diseases and also improve upon current understanding of the mechanisms of action underlying VNS.
\end{abstract}

$V^{3}$ agus nerve stimulation (VNS) is receiving increasing attention as a therapy for treating a variety of diseases (Birmingham et al. 2014). Both the cervical and subdiaphragmatic levels of the vagus nerve are potential targets for therapeutic autonomic stimulation because of ease of access, well-defined nerve trunks, direct and reflex neural pathways to thoracic and abdominal organs, as well as to the brainstem, and promising preclinical results. However, the broad-reaching innervation by the vagus nerve presents the challenge of achieving selective activation of targeted therapy-producing nerve fibers without co-activation of non-targeted fibers.

Editors: Valentin A. Pavlov and Kevin J. Tracey

Additional Perspectives on Bioelectronic Medicine available at www.perspectivesinmedicine.org

Copyright (C) 2019 Cold Spring Harbor Laboratory Press; all rights reserved; doi: 10.1101/cshperspect.a034264

Cite this article as Cold Spring Harb Perspect Med 2019;9:a034264 
E.D. Musselman et al.

VNS to treat epilepsy, depression, and obesity was approved by the Food and Drug Administration (FDA) in 1997, 2005, and 2015, respectively, although research continues to understand better the underlying mechanisms of action. Additional VNS applications being investigated include treatment of chronic heart failure (HF), tinnitus, post-traumatic stress disorder, stroke, and inflammatory diseases.

In clinical practice, the selection of stimulation parameters includes pulse width, amplitude, duty cycle, and frequency, and these choices determine which and how many nerve fibers are stimulated, as well as how frequently they are stimulated. Additional understanding of the impact of stimulation settings and revised device designs are required to activate selectively targeted nerve fibers.

In this review, consideration for the stimulation parameters used in preclinical and clinical studies of VNS for epilepsy and HF is used to highlight existing barriers to improved efficacy and tolerability and present empirically based parameter choices for improving patient outcomes.

\section{EPILEPSY}

\section{Background}

Preclinical and clinical studies of VNS for epilepsy demonstrate that stimulation parameters affect physiological activity and clinical outcomes, suggesting that manipulation of stimulation parameters can improve outcomes. Currently, $\sim 51 \%$ of patients experience a $50 \%$ or greater reduction in seizure frequency with VNS (Englot et al. 2011). However, the exceedingly large stimulation parameter space and slow feedback on changes in seizure frequency to changes in stimulation parameters make optimization for patient outcomes exceedingly challenging. Although stimulation intensity (pulse width and amplitude) is side effect limited, preclinical animal studies suggest that stimulating with a higher duty cycle and/or frequency could improve the response rate to VNS. Furthermore, evidence from preclinical studies supports the notion that delivering electrical stimulation closer to the onset of a seizure than is achieved using magnet-triggered stimulation may be more effective at aborting seizures.

\section{Treatments for Epilepsy}

Epilepsy affects over 70 million people worldwide (Singh and Trevick 2016); its prevalence does not discriminate with regard to age or sex (Fiest et al. 2017), and it is associated with a threefold increase in risk of death (Forsgren et al. 2005). Epilepsy has a profound negative impact on quality of life, general health, employment status, and income (Kobau et al. 2008).

The frontline therapy for epilepsy is antiepileptic drugs (AEDs), although a large subset of patients fails to manage their seizures with pharmacologic solutions alone. Seizures are completely eliminated in $47 \%$ of patients with the first attempted AED and in an additional $14 \%$ of patients after the second or third AED (Kwan and Brodie 2000). Refractory epilepsy, when the frequency of seizures cannot be controlled despite trying two or more AEDs, remains a major public health challenge and economic burden as $20 \%$ to $40 \%$ of all patients who are newly diagnosed with epilepsy will eventually become refractory (French 2007). For patients who fail to experience sufficient seizure reduction with pharmacologic therapies, surgical resection of epileptogenic areas can be highly effective as $52 \%$ of patients remain seizure-free 5 years postsurgery (Hirsch 2012). However, not all patients are candidates for surgical resection because of disease etiology, location of the seizure initiation zone in critical brain regions, or susceptibility to complications associated with invasive surgery (e.g., age or health status).

VNS for epilepsy was FDA-approved in 1997 as an adjunctive therapy for patients with refractory epilepsy and is used for both seizure prevention and termination. VNS for epilepsy involves implanting two helical cuff electrodes on the left cervical vagal trunk. The electrodes are connected to an implanted pulse generator placed subcutaneously in the chest. The implanted pulse generator delivers intermittent electrical stimulation, much like a pacemaker for the nerve, to evoke neural activity. The LivaNova 
(London, UK) VNS device delivers continuous stimulation, independent of epileptic activity, to reduce seizure frequency and duration. The VNS device can deliver additional stimuli when a rapid increase in heart rate (HR) is detected (ictal tachycardia), which may indicate an imminent seizure. Additionally, the VNS device features a handheld magnet and implanted sensor that allow patients or caregivers to deliver on-demand stimulation if they experience an aura, which is experienced by some patients indicating an impending seizure.

\section{Preclinical Studies of VNS for Epilepsy}

Application of VNS to treat epilepsy originated from a series of observations that VNS altered cortical synchronization in animal models. Electric potentials on the orbitofrontal cortex increased bilaterally in amplitude and rate during 24 to $50 \mathrm{~Hz}$ VNS (Bailey and Bremer 1938). Additionally, VNS eliminated or greatly reduced synchronous cortical electroencephalogram (EEG) in cats (Zanchetti et al. 1952). VNS was later demonstrated to increase or decrease EEG synchronization depending on the frequency and amplitude of stimulation (Chase et al. 1967). This study concluded that synchronization or desynchronization of neural activity is likely dependent on the group of fibers activated in the vagus nerve rather than any single stimulation parameter in isolation. These observations of VNS-induced changes in brain activity led to experiments in animal models to investigate the ability of VNS to abort chemically and electrically induced seizures.

Results in various animal models of epilepsy demonstrate that VNS is effective at aborting seizures and reducing seizure frequency, severity, and duration. In a strychnine seizure model in dogs, VNS was delivered several minutes after the initial signs of the seizure were observed in electromyography (EMG) of the gastrocnemius muscle. VNS aborted seizures within 0.5 to $5 \mathrm{sec}$ of stimulation application and also prolonged the time with no spontaneous EMG activity (Zabara 1992). In an alumina seizure model in monkeys, VNS reduced seizure frequency by stimulating the nerve at the onset of every seizure. The reduction in seizure frequency was maintained for $\sim 1$ month after the VNS was discontinued (Lockard et al. 1990). In a maximal electroshock epilepsy model in rats, VNS reduced the seizure severity and nearly eliminated muscle spasms (Woodbury and Woodbury 1990). In a penicillin/pentylenetetrazol (PTZ) model of epilepsy in rats, $20 \mathrm{sec}$ of VNS reduced interictal spike frequency by $33 \%$ and spike amplitudes also decreased. Seizure duration decreased from $\sim 30 \mathrm{sec}$ without stimulation to $\sim 5 \mathrm{sec}$ with VNS (McLachlan 1993). In an amygdala-kindling seizure model, where the amygdala was stimulated with implanted electrodes every 5 min with increasing intensity until convulsions were induced, cats treated with VNS exhibited seizure development limited to facial twitching and head nodding as compared to generalized clonic jerking and convulsion in cats without VNS (Fernandez-Guardiola et al. 1999). Promising preclinical studies results across species and models of epilepsy prompted feasibility trials of VNS as a treatment for patients with refractory epilepsy.

\section{VNS in the Clinic for Treatment of Epilepsy}

Two pilot clinical studies of VNS for epilepsy showed promising results. The E01 and E02 trials were longitudinal feasibility trials that implanted 10 and 5 patients, respectively, and compared seizure frequency before and after VNS implantation. In total, the 14 patients that completed the E01/2 trials experienced a $\sim 47 \%$ reduction in seizure frequency and $\sim 36 \%$ had a $50 \%$ or greater reduction in seizure frequency (Uthman et al. 1993). These promising initial results in feasibility trials prompted pivotal trials.

Outcomes of the multicenter, randomized, controlled E03 trial (54 patients in HIGH group, 60 patients in LOW group) and pivotal E05 trial (94 patients in HIGH group, 102 patients in LOW group) of VNS for epilepsy indicated that higher amplitudes, when used in combination with higher duty cycle, higher frequency, and longer pulse widths, were more efficacious (The Vagus Nerve Stimulation Study Group 1995; Handforth et al. 1998; DeGiorgio et al. 2000). Table 1 provides a summary of the stim- 

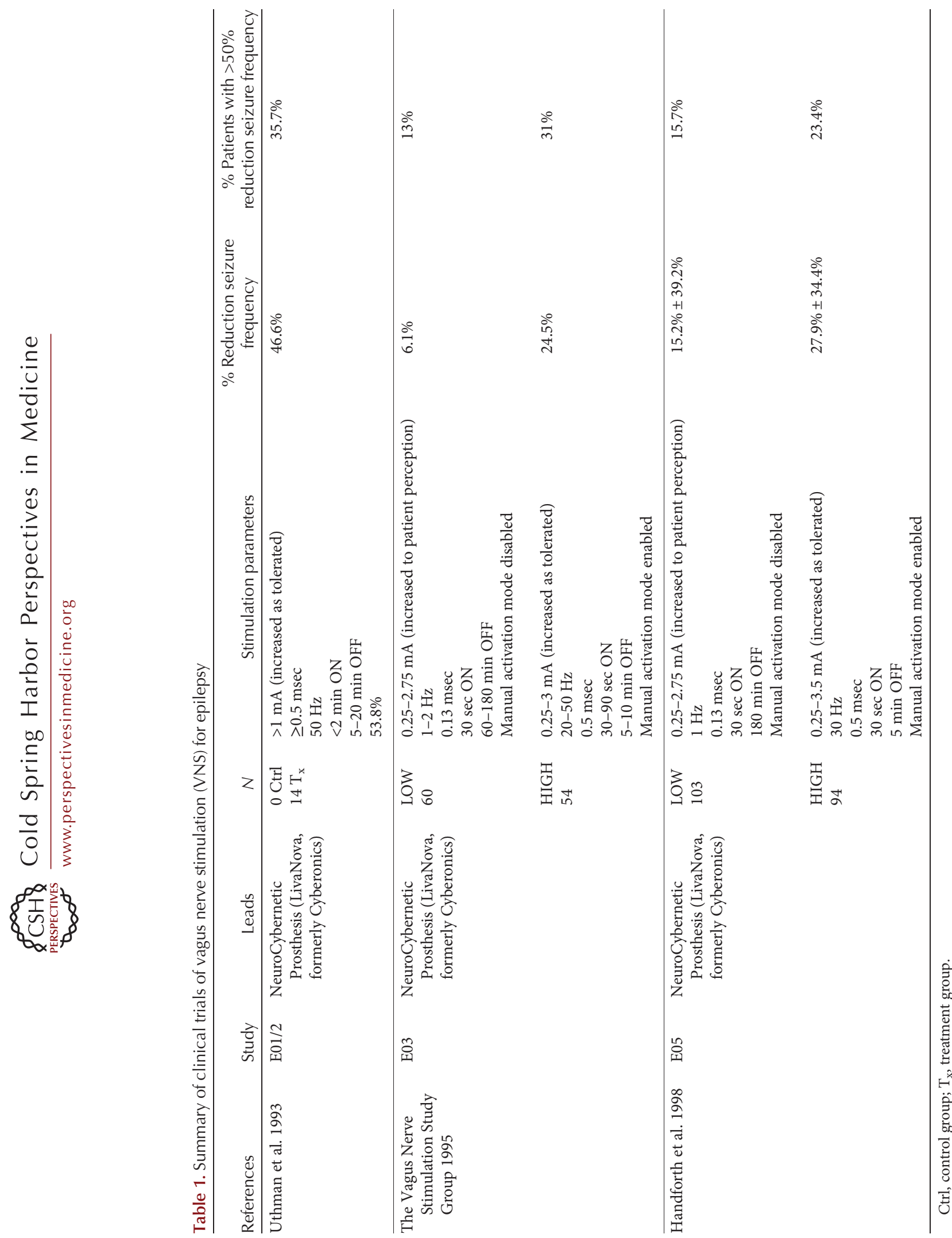
ulation methods and trial outcomes. Since stimulation of the vagus nerve is generally perceived by patients, the control group also received VNS to maintain blinding, albeit with lower duty cycle (longer OFF time), lower frequency, and shorter pulse width. Specifically, the HIGH stimulation group received 0.25 $3.5 \mathrm{~mA}$ (set to patient tolerance), $30 \mathrm{sec} \mathrm{ON}+$ $5 \mathrm{~min}$ OFF, $30 \mathrm{~Hz}$, and $500-750 \mu \mathrm{sec}$ pulse duration, while the LOW stimulation group received $0.25-2.75 \mathrm{~mA}$ (set to patient perception), $30 \mathrm{sec} \mathrm{ON}+180 \mathrm{~min}$ OFF, $1 \mathrm{~Hz}$, and $130 \mu \mathrm{sec}$ pulse duration. Additionally, only the HIGH stimulation group's device responded to the magnet that delivers on-demand stimulation to abort a seizure.

Average reduction in seizure frequency was significantly better in the $\mathrm{HIGH}$ stimulation groups than in the LOW stimulation groups, and the primary efficacy endpoints were met. Specifically, after ramping up stimulation for 2 weeks post-implantation, seizure frequency with stimulation on for 3 months was compared to a 3-month pre-operation baseline. During the final 12 weeks of therapy in the E03 trial, the HIGH stimulation group had significantly greater reduction in frequency of all seizures than the LOW stimulation group $(-24.5 \%$ vs. $-6.1 \% ; p=0.01)$. Patients in the HIGH stimulation group had a significant reduction in seizures as compared to baseline $(p<0.01 ; 95 \%$ CI, $-14.1 \%$ to $-34.9 \%$ ), whereas patients in the LOW stimulation group did not $(p=0.21$; $95 \%$ CI, $+3.6 \%$ to $-15.8 \%)$. Similarly, in the E05 trial, a statistically significant difference was achieved between the HIGH stimulation group, which achieved a $27.9 \% \pm 34.3 \%$ reduction in total seizure frequency from baseline, and the LOW stimulation group, which achieved a $15.2 \% \pm 39.2 \%$ in reduction in total seizure frequency from baseline $(p=0.02)$. At the end of the blinding period in the E03 trial, 67 patients elected to continue therapy in an unblinded extension phase. Patients in the LOW group were transitioned to $\mathrm{HIGH}$ stimulation parameters and experienced a reduction of seizure frequency comparable to the HIGH group. Accounting only for the periods when patients received $\mathrm{HIGH}$ stimulation parameters, the HIGH group and LOW to HIGH group had mean reductions in seizure frequency $\sim 43 \%$ and $\sim 38 \%$, respectively (medians $43.1 \%$ and $39.3 \%$ ), at $13-15$ months (George et al. 1994).

\section{Impact of Stimulation Parameters}

The effect of stimulation parameters on clinical outcomes was demonstrated in the E03 and E05 clinical trials in comparing the HIGH and LOW groups, but the individual contributions and interactions between amplitude, frequency, pulse width, and duty cycle were not isolated. We summarize below the effects of stimulation intensity (amplitude and pulse width), duty cycle, and frequency from preclinical studies and clinical trials of VNS to treat epilepsy.

\section{Amplitude and Pulse Width}

Although the mechanism of action of VNS for epilepsy was previously thought to involve activation of small diameter unmyelinated Cfibers (Woodbury and Woodbury 1990), this hypothesis has since been disproven in preclinical studies. Reduction of seizure intensity with VNS was maintained after C-fibers were abolished with peripheral subcutaneous injections of capsaicin in a PTZ rat model of epilepsy (Krahl et al. 2001), indicating that C-fibers were not required for the anti-epileptic effects of VNS.

In clinical studies, the stimulation intensity (i.e., combined effect of amplitude and pulse width) is too low to activate C-fibers. Stimulating the vagus nerve and recording the associated compound action potentials in canines showed that the thresholds to activate small diameter myelinated B-fibers and unmyelinated C-fibers were $\sim 10$ - and $\sim 46$-fold higher, respectively, than the threshold to activate large diameter myelinated A-fibers (Yoo et al. 2013). Since there is no VNS-induced bradycardia (reduced HR) in patients being treated for epilepsy (Banzett et al. 1999), which is generated by B-fiber activation, stimulation intensity is subthreshold for B- and C-fibers (Jones et al. 1995; Yoo et al. 2013).

The stimulation amplitude used to activate a population of nerve fibers with clinical VNS is limited by patient tolerance for a set pulse width 
E.D. Musselman et al.

and frequency. The side effects of hoarseness, coughing, and neck pain are mediated by activation of A-fibers in the vagus nerve (Banzett et al. 1999) and prevent increasing stimulation intensity (Binnie 2000). Since C-fibers have much higher thresholds than both $\mathrm{A}$ - and $\mathrm{B}$ fibers, and stimulation intensity is limited by A-fiber activation, it is extremely unlikely that any C-fibers are activated with VNS at clinical amplitudes and pulse widths. Therefore, myelinated afferents are likely the fibers responsible for anti-epileptic effects.

\section{Duty Cycle}

The duty cycle of clinical VNS for epilepsy is based on a study of PTZ-induced seizures in rats. The anticonvulsant effect of $60 \mathrm{~min}$ of VNS diminished in a time-dependent manner post-stimulation, with approximate rebound of seizure duration, total number of seizures, and number of tonic seizures by half at $5 \mathrm{~min}$ and entire loss of VNS effects at $10 \mathrm{~min}$ (Takaya et al. 1996). Further, the study showed greater reduction in seizure duration, total number of seizures, and number of tonic seizures with continuous stimulation as compared to intermittent ( $30 \mathrm{sec}$ ON, $5 \mathrm{~min}$ OFF) stimulation. These findings informed the clinical duty cycle of $30 \mathrm{sec}$ ON and $5 \mathrm{~min}$ OFF, which is a compromise between continuous stimulation and lower duty cycle to improve battery life of the device (Schachter and Saper 1998). In an unblinded continuation of the E05 study, the XE5 study, a subgroup of 26 patients refractory to VNS with conventional stimulation parameters experienced significant improvement in seizure control when the OFF time was reduced from $5 \mathrm{~min}$ to $\leq 1.1 \mathrm{~min}(21 \%$ vs. $39 \% ; p=0.011)$ and the responder rate ( $>50 \%$ reduction in seizures) also significantly improved (19\% vs. $35 \% ; p=$ 0.046). This is an important finding that suggests non-responders may receive benefit from VNS if the duty cycle was increased (DeGiorgio et al. 2001).

Recent preclinical work in rats suggests an interaction between duty cycle and stimulation amplitude in increasing VNS efficacy. In freely moving rats, Larsen and colleagues measured perforant path dentate field-evoked potentials (EPs), which are used to probe the excitability of dentate neurotransmission, a fundamental aspect of epilepsy (Larsen et al. 2016). The study found that rapid duty cycle VNS (7 sec $\mathrm{ON}+13 \mathrm{sec} \mathrm{OFF})$ at $250 \mu \mathrm{A}$ had greater effect than regular duty cycle $(30 \mathrm{sec} \mathrm{ON}+300 \mathrm{sec}$ $\mathrm{OFF})$ at $1000 \mu \mathrm{A}$, indicating that activating fewer fibers (lower stimulation amplitude) more frequently (higher duty cycle) increased the recorded neural activity. Regular duty cycle at $250 \mu \mathrm{A}$ induced no change in dentate field EP parameters, indicating that higher duty cycle and/or stimulation amplitude are required for changes in the neural output measure.

\section{Frequency}

The pulse repetition frequency of VNS for epilepsy was chosen based on studies of seizure suppression in dogs, which indicated that within the tested range of $20-150 \mathrm{~Hz}$ the optimal range is $20-30 \mathrm{~Hz}$ for interrupting or abolishing strychnine-induced seizures and PTZ-induced tremors (Zabara 1992). This finding was supported by preclinical studies in rats suggesting that within the tested range of $1-20 \mathrm{~Hz}$, 10-20 Hz shortened 3-mercaptopropionate- or PTZ-induced seizures (Woodbury and Woodbury 1990). However, in a PTZ-induced seizure model in rats, 130 and $180 \mathrm{~Hz}$ VNS generated a $50 \%$ larger attenuation of seizures than $30 \mathrm{~Hz}$ VNS, as measured by normalized mean power and frequency from intracortical recordings of spike-and-wave discharges (Jiao et al. 2016). If additional experiments in different seizure animal models show promise for therapeutic improvements from 130 and $180 \mathrm{~Hz}$ VNS, clinical trials may be conducted to confirm translation of effects.

\section{Closed-Loop Stimulation}

Although clinical VNS is typically delivered continuously in an open-loop fashion (typically $30 \mathrm{sec} \mathrm{ON}+5 \mathrm{~min}$ OFF, $24 \mathrm{~h} /$ day) for seizure prevention, acute delivery of additional stimulation (30 sec ON period) can abort an imminent seizure detected by an aura or increase in HR 
(i.e., behaviorally vs. physiologically controlled closed-loop stimulation, respectively). In patients with generalized epilepsy, $\sim 64 \%$ of patients experience at least one form of aura (Spencer 2015). Studies with simultaneous EEG and electrocardiogram (ECG) recordings demonstrated a significant increase in HR with ictal events in $82 \%$ of epilepsy patients (Eggleston et al. 2014).

Whereas epilepsy patients or caregivers can use the handheld magnet to deliver additional stimulation when an aura is detected, the efficacy of this VNS delivery method is unclear. For some patients, on-demand stimulation helps terminate seizures and decreases the intensity or duration of the seizures (Fisher et al. 2015). In the E03 clinical trial, $21 \%$ of seizures in the active treatment group (HIGH, magnet on) and $\sim 9 \%$ of seizures in the control group (LOW, magnet inactive) were aborted with magnet-activated stimulation (Ben-Menachem et al. 1994). However, in the E05 clinical trial, there was no difference in the reduction of seizure frequency in patients with or without auras when they had control of the magnet mode (Handforth et al. 1998).

More recent developments of the VNS implant provide a closed-loop system to monitor $\mathrm{HR}$, where a rapid increase (i.e., tachycardia) may indicate an imminent seizure, thereby triggering additional acute stimulation to try to abort the seizure or reduce seizure severity (Boon et al. 2015; Hampel et al. 2015). When VNS is delivered earlier in the time course of a seizure, it is more effective at reducing seizure duration (McLachlan 1993). In this study of rats treated with PTZ, if VNS was delivered $>4 \mathrm{sec}$ after seizure onset, there was no observed changed in seizure duration. However, stimulating $<3 \mathrm{sec}$ after seizure onset reduced the mean seizure duration from $\sim 30 \mathrm{sec}$ without stimulation to $\sim 5 \mathrm{sec}$ with stimulation. Twenty patients with ictal tachycardia were implanted with AspireSR (LivaNova, London, UK) and treated with AutoStim VNS in the U.S. E-37 trial. They experienced seizure termination (i.e., when seizures ended before the $60 \mathrm{sec}$ course of AutoStim concluded) $\sim 61 \%$ of the time across all seizure types. Within this group, simple partial seizures terminated with AutoStim were $\sim 62 \%$ shorter (32 sec with AutoStim vs. $85 \mathrm{sec}$ historically) and complex partial seizures were $\sim 8 \%$ shorter (74 sec with AutoStim vs. $80 \mathrm{sec}$ historically) from the patient's baseline of historical seizures without AutoStim (Fisher et al. 2016). In aggregate, median time between seizure onset and termination with AutoStim was reduced to $35 \mathrm{sec}$. For seizures terminated during AutoStim, shorter latency between seizure onset and initiation of AutoStim corresponded to shorter seizure duration.

\section{Future Work}

Improved clinical outcomes from VNS for epilepsy will require determining how stimulation parameters affect the population of nerve fibers that are stimulated and a more specific understanding of the underlying mechanisms of action of VNS. Animal studies and clinical data suggest that stimulating with a higher duty cycle and/or higher frequency may improve the response rate to VNS. Animal studies also demonstrated that stimulation closer to the onset of a seizure is more effective at aborting seizures, supporting the use of closed-loop systems.

\section{HEART FAILURE}

\section{Background}

VNS for treatment of HF showed therapeutic benefits in animal models, but multiple clinical trials failed to meet their primary endpoints. The lack of successful translation of preclinical results to the clinic is likely a result of inadequate activation of the nerve fibers that produce therapeutic effect. Large diameter myelinated A-fibers in the vagus nerve innervate neck muscles and have lower thresholds than the thinly myelinated efferent B-fibers that have therapeutic effects for treating HF and cause bradycardia (i.e., reduction in HR) (Yoo et al. 2013). In animal models of HF, VNS current amplitude was increased until bradycardia was induced (Vanoli et al. 1991; Li et al. 2004; Sabbah et al. 2005; Zhang et al. 2009). In contrast, in clinical trials, the upper limit of current amplitude was re- 
E.D. Musselman et al.

stricted to limit activation of vagal motor fibers causing unwanted side effects of laryngeal contractions, and, at most, modest bradycardia was induced (De Ferrari et al. 2011; Premchand et al. 2014; Zannad et al. 2015; Gold et al. 2016). Since B-fibers have substantially higher activation thresholds than the laryngeal motor fibers, titration of amplitude to limit side effects produced by A-fiber activation likely resulted in inadequate therapeutic efficacy.

$\mathrm{HF}$ is a disease that affects over 26 million people worldwide (Ponikowski et al. 2014). Primarily due to the aging population, predictions indicate a 46\% increase from 2012 to 2030 in the number of people living in the United States with HF (Heidenreich et al. 2013). HF patients have a 1-year mortality rate of about 50\% (Cleland et al. 1999), and by 2030, HF will cost the U.S. economy $\$ 53$ billion in direct medical costs and $\$ 17$ billion in indirect costs per year (Heidenreich et al. 2013). For many patients, HF symptoms are not adequately controlled with drugs, as evidenced by high rates of repeat hospitalizations. HF becomes progressively more severe and there is a significant unmet clinical need for innovative therapies in this patient population.

The progression of $\mathrm{HF}$ is associated with characteristic changes in autonomic activity in the vagus nerve. Poor clinical outcomes for HF patients correspond to increased sympathetic activity, decreased parasympathetic (vagal) activity, and loss of HR variability (Mortara et al. 1997; Ponikowski et al. 1997). Pharmacologic agents, particularly $\beta$-adrenergic agonists, are an effective treatment for systolic HF (Hernandez et al. 2009). Second-line treatments for HF include cardiac resynchronization therapy, left ventricular (LV) assist devices, and heart transplant (Lyons et al. 2017).

$\mathrm{HF}$ with a reduced ejection fraction (HFrEF), also termed systolic HF, is responsible for $53 \%$ of all HF cases (Owan et al. 2006). VNS to treat HFrEF may offer an alternative to pharmacologic treatments. Although studies in rat and canine animal models show improved heart function and increased survival, clinical trials repeatedly failed to demonstrate comparable therapeutic benefits.

\section{Preclinical Studies of VNS for HF}

In preclinical studies, stimulation-induced bradycardia, indicating activation of B-fibers (Yoo et al. 2013), was used to select stimulation amplitudes, although the resulting amplitudes varied between individual animals, species, stimulation waveforms, electrode designs, electrode locations, anesthesia, and experimental setups. Table 2 presents a summary of the stimulation methods, experimental HF models, and study outcomes. VNS increased survival rates in a rat model of HF induced by ligating the left coronary artery to mimic a myocardial infarction (Li et al. 2004). In the treatment group, the right cervical vagus nerve was stimulated at $20 \mathrm{~Hz}$ with a pulse width of $0.2 \mathrm{msec}$; the current was increased to reduce HR by $20-30 \mathrm{bpm}$ ( $5 \%$ $10 \%$ reduction from resting $\mathrm{HR})$. The results showed improved heart function: lower end-diastolic pressure (17.1 \pm 5.9 vs. $23.5 \pm 4.2 \mathrm{mmHg}$; $p<0.05)$, higher rate of change of the LV pressure with time $(4152 \pm 237$ vs. $2987 \pm 192 \mathrm{mmHg} /$ sec; $p<0.05)$, and decreased normalized biventricular weight $(2.75 \pm 0.25$ vs. $3.14 \pm 0.22$ $\mathrm{g} / \mathrm{kg} ; p<0.01)$. Additionally, VNS provided a significant increase in 20 -week survival $(86 \%$ vs. $50 \% ; p=0.008$ )

In canine models of HF, VNS produces antiarrhythmic effects, favorable impact on heart function and LV remodeling (change in heart muscle structure resulting from injury), and protective effects against sudden cardiac death (Vanoli et al. 1991; Sabbah et al. 2005, 2008; Zhang et al. 2009). In a canine microembolization-induced HF model, right cervical VNS at an amplitude that produced a $10 \%$ reduction in HR resulted in significantly improved LV systolic and diastolic function (Sabbah et al. 2005, 2008). These results suggest that the stimulation intensity was sufficient to activate the bradycardia-inducing B-fibers.

In a canine healed anterior myocardial infarction model of HF, right cervical VNS effectively prevented sudden cardiac death: $87 \%$ of the control group developed ventricular fibrillation (VF), compared to only $10 \%$ of the treatment group $(p<0.001)$ (Vanoli et al. 1991). Further, the HR in the VNS group was $170 \pm$ 
Selecting Vagus Nerve Stimulation Parameters
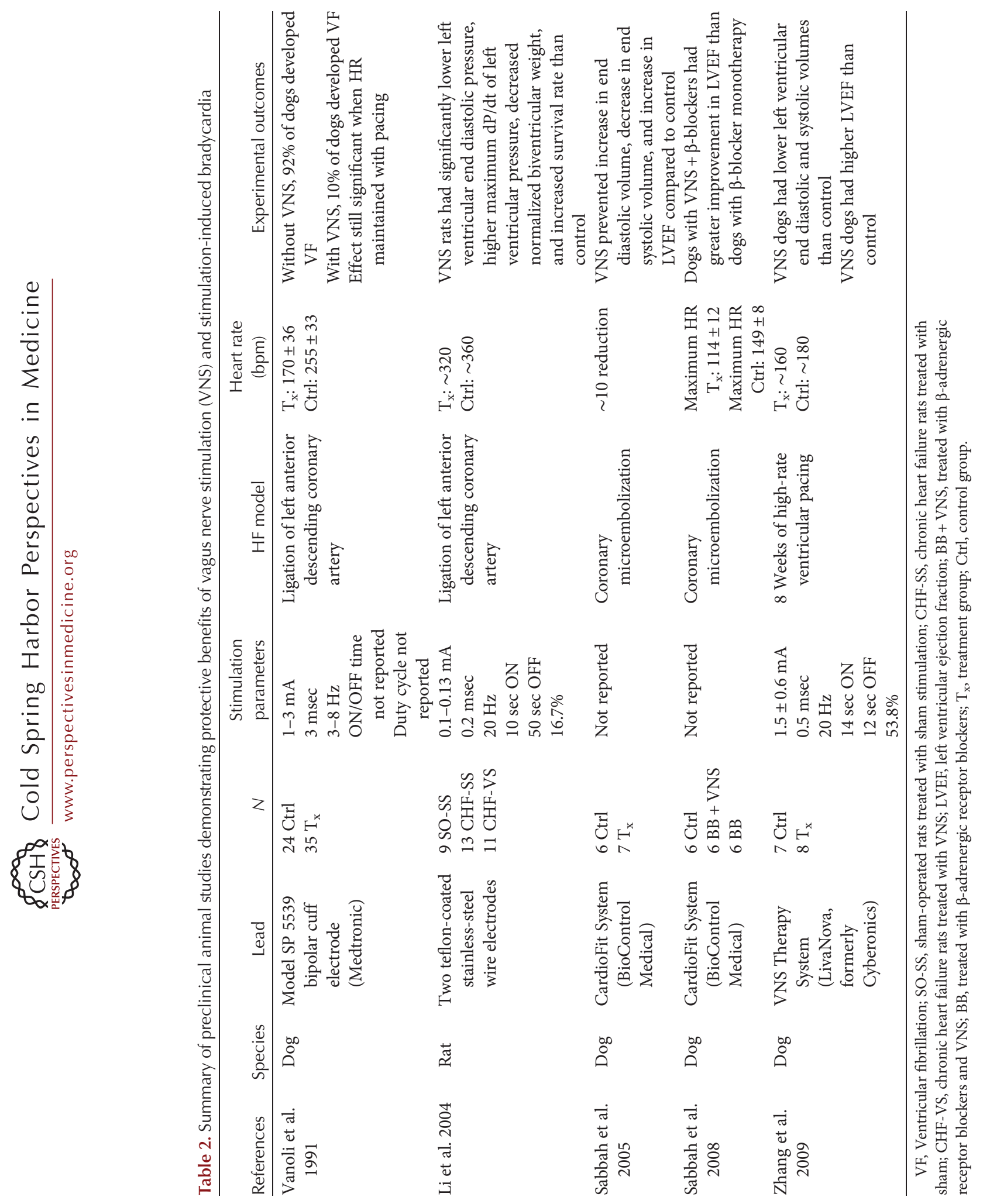
E.D. Musselman et al.

36 beats per minute compared to $255 \pm 33$ beats per minute in the control condition $(p<0.001)$. To isolate whether the protective effects of VNS were mediated simply by the reduction in $\mathrm{HR}$, pacing to the atrium was applied simultaneously with VNS therapy, and the protection in the treatment group with VNS was still significant $(p=0.015)$. These findings indicate that antiarrhythmic benefits are segregated from the reduction in HR (Ardell et al. 2017). This implies that the therapeutic fibers share a common excitability (i.e., fiber diameter) with bradycardia-inducing fibers, although they may not necessarily be the same population of fibers.

Collectively, the preclinical studies of VNS for HF demonstrated that using stimulation parameters that induced moderate bradycardia (activation of B-fibers) was correlated with improved heart function, increased survival, antiarrhythmic affects, and had a favorable impact on heart remodeling after infarction, independent of the reduction in HR alone.

\section{VNS in the Clinic for Treatment of Heart Failure}

A series of clinical trials for HF recruited similar patient populations but achieved inconsistent clinical outcomes. In the CardioFit Pilot, INOVATE-HF, NECTAR-HF, and ANTHEMHF studies, the patients all met similar criteria, including NYHA class II-III/IV HF and left ventricular ejection fraction (LVEF) less than 35\% or $40 \%$. Table 3 provides a summary of the stimulation methods and trial outcomes.

The CardioFit Pilot of VNS for HF enrolled 32 patients with a history of chronic HF and showed promising results (De Ferrari et al. 2011). The CardioFit system (BioControl Medical) included a cuff electrode placed around the right cervical $\mathrm{VN}$ and used a right ventricular sensing lead to provide closed-loop control. The patients enrolled in this study were already receiving standard medical treatment for $\mathrm{HF}$ with pharmacologic therapies, including $\beta$-blockers, angiotensin-converting enzyme (ACE) inhibitors, angiotensin II receptor blockers, and loop diuretics; 19 were also implanted with an implanted cardioverter defibrillator. Stimulation was delivered at the highest tolerable amplitude $(4.1 \pm 1.2 \mathrm{~mA})$. The VNS-induced bradycardia, reducing HR by $7.3 \pm 3 \mathrm{bpm}$. The patients experienced improvements in quality-of-life metrics, as well as significant reduction in LV end systolic volume and increase in LVEF (from $22 \% \pm 7 \%$ to $29 \% \pm 8 \%$ ). Improvements in patient condition were maintained at 1 and 2 years.

The INOVATE-HF phase III multicenter randomized clinical trial used the CardioFit system (BioControl Medical) to follow-up on the CardioFit pilot study (Gold et al. 2016). The study was designed to assess the safety and efficacy of VNS for HF in 707 patients with symptomatic HF despite receiving optimal medical therapy (Hauptman et al. 2012; Gold et al. 2016). The study was terminated early when it became clear that it would not meet its primary efficacy endpoint of statistically significant reduction of death from any cause or first event for worsening HF. When the study was terminated, the mean follow-up period was 16 months. At this time, the primary efficacy endpoint occurred in 132 of 436 patients (30.3\%) in the VNS group compared with 70 of 271 $(25.8 \%)$ in the control group $(p=0.37)$.

In the double-blinded, randomized NECTAR-HF clinical trial, the Precision system (Boston Scientific) delivered open-loop bipolar stimulation for patients in the treatment group; patients in the control group received an implant but no stimulation (Zannad et al. 2015). The stimulation current was again set to the maximum tolerable amplitude $(1.42 \pm 0.80 \mathrm{~mA})$. The primary efficacy endpoint required a significantly different change in LV end-systolic diameter from baseline between the treatment and control groups at 6 months, but this target was not attained $(4.9 \pm 0.8 \mathrm{~cm}$ with VNS vs. $5.1 \pm$ $0.8 \mathrm{~cm}$ for control; $p=0.60$ ). Consistent with previous data suggesting that VNS must cause bradycardia to indicate that the nerve fibers producing the therapeutic effects are indeed being activated, 24-h ECG monitoring with a Holter monitor showed no significant reduction in maximum, minimum, or mean HR in the treatment group between baseline and 6 months.

The 60 patients in the ANTHEM-HF trial were implanted with the Cyberonics Model 103 

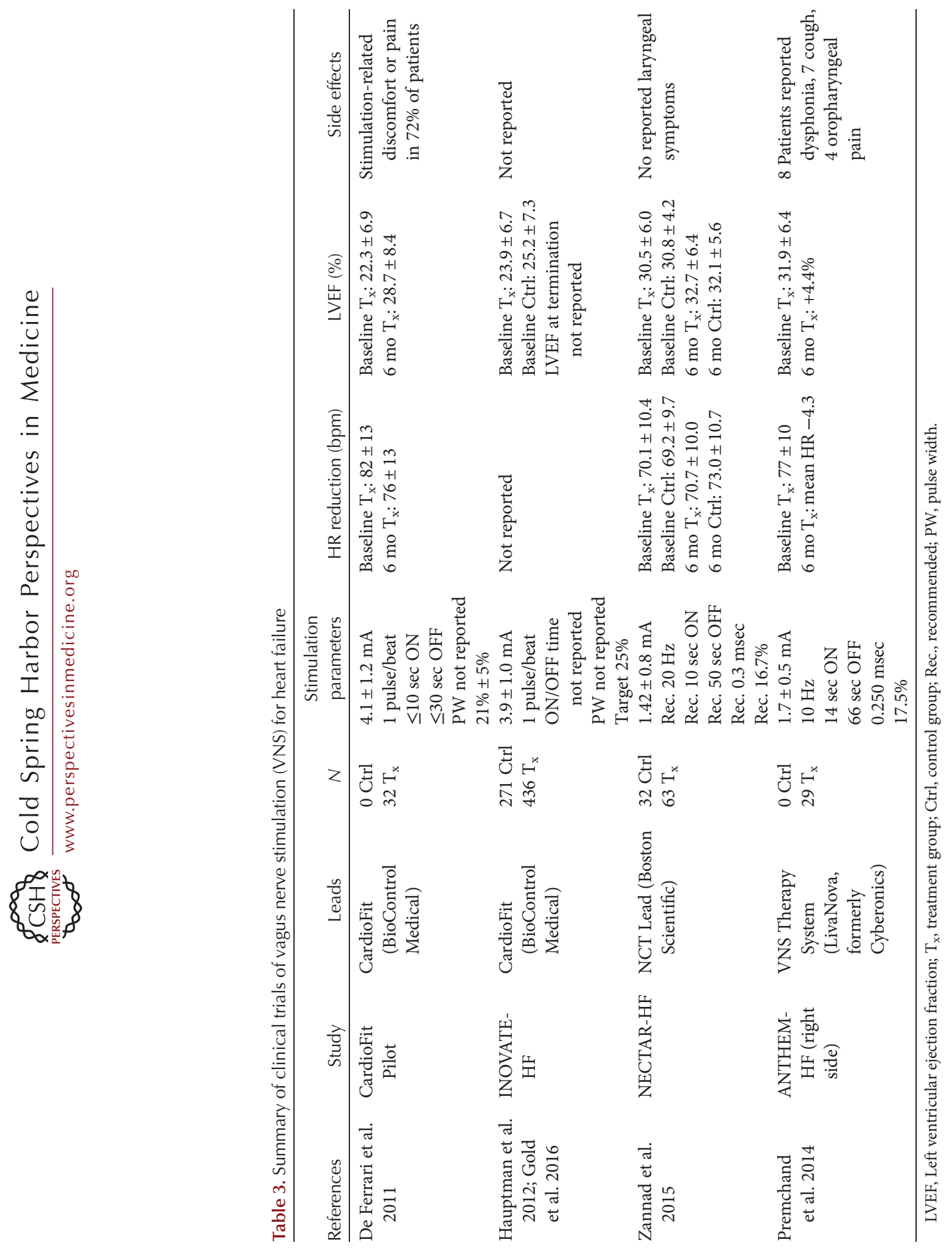
E.D. Musselman et al.

system and received bipolar stimulation on the left or right cervical VN using the highest tolerable stimulation amplitude $(1.7 \pm 0.5 \mathrm{~mA}$ right side, $2.2 \pm 0.5 \mathrm{~mA}$ left side) (Dicarlo et al. 2013; Premchand et al. 2014). The defined primary efficacy objective was to evaluate the effects of chronic VNS on echocardiographic changes in LV end-systolic volume and LVEF. LVEF increased by $4.5 \%$ (95\% CI $2.4 \%$ to $6.6 \%$ ), qualityof-life scores improved, yet 24-h Holter monitor ECG recording demonstrated no decrease in $\mathrm{HR}$ from baseline to 6 months.

In animal models and in clinical studies, increasing stimulation intensity to produce bradycardia (activation of B-fibers) appears to be a consistent biomarker for therapeutic benefit in systolic HF, suggesting that bradycardia and therapeutic benefit are mediated by nerve fibers with similar excitability. However, across species, different levels of A-fiber activation appear to be tolerated as evidenced by significantly higher degrees of bradycardia achieved in dogs and rats than in humans. VNS-induced bradycardia is mediated by B-fiber activation, which requires a $\sim 10$-fold increase in threshold amplitude over A-fibers (Yoo et al. 2013). Thus, to induce bradycardia, a robust activation of A-fibers will also occur as a result of activation of larger diameter nerve fibers at lower currents than smaller diameter fibers.

In Vanoli and colleagues' canine experiments, in which VNS reduced HR by $\sim 33 \%$ from control $(255 \pm 33 \mathrm{bpm}$ to $170 \pm 36 \mathrm{bpm})$ after $30 \mathrm{sec}$ of occlusion during exercise, the stimulation parameters selected to achieve bradycardia resulted in coughing for about one-third of tests and retching in a few cases. However, it was never perceived that the animals were in pain (Vanoli et al. 1991). In clinical use, stimulation intensity is determined by increasing the amplitude to the highest tolerable current amplitude. Of the 60 patients enrolled in the ANTHEM-HF trial, 19 (31.7\%) of patients reported dysphonia, $13(21.7 \%)$ reported cough, and eight (13.3\%) reported oropharyngeal pain (Premchand et al. 2014). Patients in the clinic experience intolerable side effects attributed to activation of motor A-fibers to the larynx at intensities lower than required to produce brady- cardia and activate the nerve fibers that appear to mediate therapeutic benefit.

\section{CONCLUDING REMARKS}

Although the mid-cervical level of the vagus nerve is a convenient surgical access point to implant VNS electrodes, broad-reaching innervation to a diverse range of organ targets makes selective stimulation of targeted nerve fibers without co-activation of non-targeted nerve fibers a great challenge. Improved understanding of the mechanisms of action behind VNS to treat epilepsy and HF and the stimulation parameters best-suited to selectively activate nerve fibers is essential to improving clinical outcomes and expanding VNS to novel applications. Experimental studies and validated computational models will be essential in answering these fundamental questions.

\section{ACKNOWLEDGMENTS}

Preparation of this review was supported in part by National Institutes of Health $(\mathrm{NIH})$ OT2 OD025340.

\section{REFERENCES}

Ardell JL, Nier H, Hammer M, Southerland EM, Ardell CL, Beaumont E, KenKnight BH, Armour JA. 2017. Defining the neural fulcrum for chronic vagus nerve stimulation: Implications for integrated cardiac control. J Physiol 595: 6887-6903.

Bailey P, Bremer F. 1938. A sensory cortical representation of the vagus nerve: With a note on the effects of low blood pressure on the cortical electrogram. J Neurophysiol 1: 405-412.

Banzett RB, Guz A, Paydarfar D, Shea SA, Schachter SC, Lansing RW. 1999. Cardiorespiratory variables and sensation during stimulation of the left vagus in patients with epilepsy. Epilepsy Res 35: 1-11.

Ben-Menachem E, Mañon-Espaillat R, Ristanovic R, Wilder BJ, Stefan H, Mirza W, Tarver WB, Wernicke JF; First International Vagus Nerve Stimulation Study Group. 1994. Vagus nerve stimulation for treatment of partial seizures. 1: A controlled study of effect on seizures. Epilepsia 35: 616-626.

Binnie CD. 2000. Vagus nerve stimulation for epilepsy: A review. Seizure 9: 161-169.

Birmingham K, Gradinaru V, Anikeeva P, Grill WM, Pikov V, Weber D, McLaughlin B, Pasricha P, Ludwig K, Famm K. 2014. Bioelectronic medicines: A research roadmap. Nat Rev Drug Discov 13: 399-400. 
Boon P, Vonck K, van Rijckevorsel K, Tahry RE, Elger CE, Mullatti N, Schulze-Bonhage A, Wagner L, Diehl B, Hamer H, et al. 2015. A prospective, multicenter study of cardiac-based seizure detection to activate vagus nerve stimulation. Seizure 32: 52-61.

Chase MH, Nakamura Y, Clemente CD, Sterman MB. 1967. Afferent vagal stimulation: Neurographic correlates of induced EEG synchronization and desynchronization. Brain Res 5: 236-249.

Cleland JG, Gemmell I, Khand A, Boddy A. 1999. Is the prognosis of heart failure improving? Eur J Heart Fail 1: 229-241.

De Ferrari GM, Crijns HJGM, Borggrefe M, Milasinovic G, Smid J, Zabel M, Gavazzi A, Sanzo A, Dennert R, Kuschyk J; CardioFit Multicenter Trial Investigators. 2011. Chronic vagus nerve stimulation: A new and promising therapeutic approach for chronic heart failure. Eur Heart $J$ 32: $847-855$

DeGiorgio CM, Schachter SC, Handforth A, Salinsky M, Thompson J, Uthman B, Reed R, Collins S, Tecoma E, Morris GL, et al. 2000. Prospective long-term study of vagus nerve stimulation for the treatment of refractory seizures. Epilepsia 41: 1195-1200.

DeGiorgio CM, Thompson J, Lewis P, Arrambide S, Naritoku D, Handforth A, Labar D, Mullin P, Heck C; The VNS U.S. Study Group. 2001. Vagus nerve stimulation: Analysis of device parameters in 154 patients during the long-term XE5 study. Epilepsia 42: 1017-1020.

Dicarlo L, Libbus I, Amurthur B, KenKnight BH, Anand IS. 2013. Autonomic regulation therapy for the improvement of left ventricular function and heart failure symptoms: The ANTHEM-HF study. J Card Fail 19: 655-660.

Eggleston KS, Olin BD, Fisher RS. 2014. Ictal tachycardia: The head-heart connection. Seizure 23: 496-505.

Englot DJ, Chang EF, Auguste KI. 2011. Vagus nerve stimulation for epilepsy: A meta-analysis of efficacy and predictors of response. J Neurosurg 115: 1248-1255.

Fernandez-Guardiola A, Martinez A, Valdes-Cruz A, Magdaleno-Madrigal VM, Martinez D, Fernandez-Mas R. 1999. Vagus nerve prolonged stimulation in cats: Effects on epileptogenesis (amygdala electrical kindling): Behavioral and electrographic changes. Epilepsia 40: 822-829.

Fiest KM, Sauro KM, Wiebe S, Patten SB, Kwon CS, Dykeman J, Pringsheim T, Lorenzetti DL, Jetté N. 2017. Prevalence and incidence of epilepsy. Neurology 88: 296.

Fisher RS, Eggleston KS, Wright CW. 2015. Vagus nerve stimulation magnet activation for seizures: A critical review. Acta Neurol Scand 131: 1-8.

Fisher RS, Afra P, Macken M, Minecan DN, Bagić A, Benbadis SR, Helmers SL, Sinha SR, Slater J, Treiman D, et al 2016. Automatic vagus nerve stimulation triggered by ictal tachycardia: Clinical outcomes and device performance-The U.S. E-37 trial. Neuromodulation 19: 188195.

Forsgren L, Hauser WA, Olafsson E, Sander JWAS, Sillanpaa M, Tomson T. 2005. Mortality of epilepsy in developed countries: A review. Epilepsia 46: 18-27.

French JA. 2007. Refractory epilepsy: Clinical overview. Epilepsia 48: 3-7.

George R, Salinsky M, Kuzniecky R, Rosenfeld W, Bergen D, Tarver WB, Wernicke JF; First International Vagus Nerve
Stimulation Study Group. 1994. Vagus nerve stimulation for treatment of partial seizures. 3: Long-term follow-up on first 67 patients exiting a controlled study. Epilepsia 35: 637-643.

Gold MR, Veldhuisen DJV, Hauptman PJ, Borggrefe M, Kubo SH, Lieberman RA, Milasinovic G, Berman BJ, Djordjevic S, Neelagaru S, et al. 2016. Vagus nerve stimulation for the treatment of heart failure: The INOVATEHF trial. J Am Coll Cardiol 68: 149-158.

Hampel KG, Vatter H, Elger CE, Surges R. 2015. Cardiacbased vagus nerve stimulation reduced seizure duration in a patient with refractory epilepsy. Seizure 26: 81-85.

Handforth A, DeGiorgio CM, Schachter SC, Uthman BM, Naritoku DK, Tecoma ES, Henry TR, Collins SD, Vaughn BV, Gilmartin RC, et al. 1998. Vagus nerve stimulation therapy for partial-onset seizures: A randomized activecontrol trial. Neurology 51: 48-55.

Hauptman PJ, Schwartz PJ, Gold MR, Borggrefe M, Van Veldhuisen DJ, Starling RC, Mann DL. 2012. Rationale and study design of the increase of vagal tone in heart failure study: INOVATE-HF. Am Heart J 163: 954-962.

Heidenreich PA, Albert NM, Allen LA, Bluemke DA, Butler J, Fonarow GC, Ikonomidis JS, Khavjou O, Konstam MA, Maddox TM, et al. 2013. Forecasting the impact of heart failure in the United States: A policy statement from the American Heart Association. Circ Heart Fail 6: 606-619.

Hernandez AF, Hammill BG, O'Connor CM, Schulman KA, Curtis LH, Fonarow GC. 2009. Clinical effectiveness of $\beta$ blockers in heart failure: Findings from the OPTIMIZEHF (organized program to initiate lifesaving treatment in hospitalized patients with heart failure) registry. J Am Coll Cardiol 53: 184-192.

Hirsch LJ. 2012. Long-term outcome after epilepsy surgery: Relapsing, remitting disorder? Epilepsy Curr 12: 140-142.

Jiao J, Harreby K, Sevencu C, Jensen W. 2016. Optimal vagus nerve stimulation frequency for suppression of spikeand-wave seizures in rats. Artif Organs 40: 120-127.

Jones JF, Wang Y, Jordan D. 1995. Heart rate responses to selective stimulation of cardiac vagal $\mathrm{C}$ fibres in anaesthetized cats, rats and rabbits. J Physiol 489: 203-214.

Kobau R, Zahran H, Thurman DJ, Zack MM, Henry TR, Schachter SC, Price PH. 2008. Epilepsy surveillance among adults-19 States, behavioral risk factor surveillance system, 2005. MMWR Surveill Summ 57: 1-20.

Krahl SE, Senanayake SS, Handforth A. 2001. Destruction of peripheral c-fibers does not alter subsequent vagus nerve stimulation-induced seizure suppression in rats. Epilepsia 42: 586-589.

Kwan P, Brodie MJ. 2000. Early identification of refractory epilepsy. N Engl J Med 342: 314-319.

Larsen LE, Wadman WJ, van Mierlo P, Delbeke J, Grimonprez A, Van Nieuwenhuyse B, Portelli J, Boon P, Vonck K, Raedt R. 2016. Modulation of hippocampal activity by vagus nerve stimulation in freely moving rats. Brain Stimul 9: 124-132.

Li M, Zheng C, Sato T, Kawada T, Sugimachi M, Sunagawa K. 2004. Vagal nerve stimulation markedly improves longterm survival after chronic heart failure in rats. Circulation 109: 120-124. 
E.D. Musselman et al.

Lockard JS, Congdon WC, DuCharme LL. 1990. Feasibility and safety of vagal stimulation in monkey model. Epilepsia 31: 20-26.

Lyons KJ, Ezekowitz JA, Liang L, Heidenrich PA, Yancy CW, Devore AD, Hernandez AF, Fonarow GC. 2017. The impact of the current versus prior cardiac resynchronization therapy guidelines on the proportion of the heart failure patients eligible for therapy. JACC: Heart Failure 5: 388392.

McLachlan RS. 1993. Suppression of interictal spikes and seizures by stimulation of the vagus nerve. Epilepsia 34: 918-923.

Mortara A, La Rovere MT, Pinna GD, Prpa A, Maestri R, Febo O, Pozzoli M, Opasich C, Tavazzi L. 1997. Arterial baroreflex modulation of heart rate in chronic heart failure: Clinical and hemodynamic correlates and prognostic implications. Circulation 96: 3450-3458.

Owan TE, Hodge DO, Herges RM, Jacobsen SJ, Roger VL, Redfield MM. 2006. Trends in prevalence and outcome of heart failure with preserved ejection fraction. $N$ Engl J Med 355: 251-259.

Ponikowski P, Anker SD, Chua TP, Szelemej R, Piepoli M, Adamopoulos S, Webb-Peploe K, Harrington D, Banasiak W, Wrabec K, et al. 1997. Depressed heart rate variability as an independent predictor of death in chronic congestive heart failure secondary to ischemic or idiopathic dilated cardiomyopathy. Am J Cardiol 79: 16451650.

Ponikowski P, Anker SD, AlHabib KF, Cowie MR, Force TL, Hu S, Jaarsma T, Krum H, Rastogi V, Rohde LE, et al. 2014. Heart failure: Preventing disease and death worldwide: Addressing heart failure. ESC Heart Fail 1: 4-25.

Premchand RK, Sharma K, Mittal S, Monteiro R, Dixit S, Libbus I, DiCarlo LA, Ardell JL, Rector TS, Amurthur B, et al. 2014. Autonomic regulation therapy via left or right cervical vagus nerve stimulation in patients with chronic heart failure: Results of the ANTHEM-HF Trial. J Card Fail 20: 808-816.

Sabbah HN, Rastogi S, Mishra S, Gupta RC, Ilsar I, Imai M, Cohen U, Ben-David T, Ben-Ezra O. 2005. 744 Long-term therapy with neuroselective electric vagus nerve stimulation improves LV function and attenuates global LV remodelling in dogs with chronic heart failure. Eur J Heart Fail Suppl 4: 166-167.

Sabbah HN, Imai M, Zaretsky A, Rastogi S, Wang M, Jiang A, Zaca V. 2008. 509 Therapy with vagus nerve electrical stimulation combined with $\beta$-blockade improves left ventricular systolic function in dogs with heart failure beyond that seen with $\beta$-blockade alone. Eur J Heart Fail Suppl 6: 114-114.

Schachter SC, Saper CB. 1998. Vagus nerve stimulation. Epilepsia 39: 677-686.

Singh A, Trevick S. 2016. The epidemiology of global epilepsy. Neurol Clin 24: 837-847.

Spencer D. 2015. Auras are frequent in patients with generalized epilepsy. Epilepsy Curr 15: 75-77.

Takaya M, Terry WJ, Naritoku DK. 1996. Vagus nerve stimulation induces a sustained anticonvulsant effect. Epilepsia 37: 1111-1116.

The Vagus Nerve Stimulation Study Group. 1995. A randomized controlled trial of chronic vagus nerve stimulation for treatment of medically intractable seizures. $\mathrm{Neu}$ rology 45: 224-230.

Uthman BM, Wilder BJ, Penry JK, Dean C, Ramsay RE, Reid SA, Hammond EJ, Tarver WB, Wernicke JF. 1993. Treatment of epilepsy by stimulation of the vagus nerve. $\mathrm{Neu}$ rology 43: 1338-1345.

Vanoli E, Ferrari GMD, Stramba-Badiale M, Hull SS, Foreman RD, Schwartz PJ. 1991. Vagal stimulation and prevention of sudden death in conscious dogs with a healed myocardial infarction. Circ Res 68: 1471-1481.

Woodbury DM, Woodbury JW. 1990. Effects of vagal stimulation on experimentally induced seizures in rats. Epilepsia 31: S7-S19.

Yoo PB, Lubock NB, Hincapie JG, Ruble SB, Hamann JJ, Grill WM. 2013. High-resolution measurement of electrically evoked vagus nerve activity in the anesthetized dog. J Neural Eng 10: 026003.

Zabara J. 1992. Inhibition of experimental seizures in canines by repetitive vagal stimulation. Epilepsia 33: 1005-1012.

Zanchetti A, Wang SC, Moruzzi G. 1952. The effect of vagal afferent stimulation on the EEG pattern of the cat. Electroencephalogr Clin Neurophysiol 4: 357-361.

Zannad F, De Ferrari GM, Tuinenburg AE, Wright D, Brugada J, Butter C, Klein H, Stolen C, Meyer S, Stein K, et al. 2015. Chronic vagal stimulation for the treatment of low ejection fraction heart failure: Results of the neural cardiac therapy for heart failure (NECTAR-HF) randomized controlled trial. Eur Heart J 36: 425-433.

Zhang Y, Popović ZB, Bibevski S, Fakhry I, Sica DA, Wagoner DRV, Mazgalev TN. 2009. Chronic vagus nerve stimulation improves autonomic control and attenuates systemic inflammation and heart failure progression in a canine high-rate pacing model. Circ Heart Fail 2: 692699. 


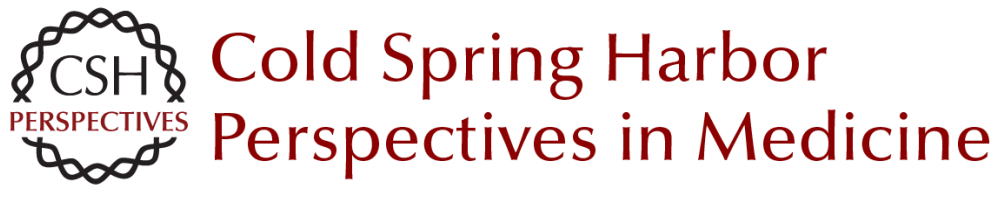

\title{
Empirically Based Guidelines for Selecting Vagus Nerve Stimulation Parameters in Epilepsy and Heart Failure
}

\author{
Eric D. Musselman, Nicole A. Pelot and Warren M. Grill
}

Cold Spring Harb Perspect Med 2019; doi: 10.1101/cshperspect.a034264 originally published online September 4, 2018

\section{Subject Collection Bioelectronic Medicine}

Neural Control of Inflammation: Bioelectronic Medicine in Treatment of Chronic Inflammatory Disease

Michael Eberhardson, Laura Tarnawski, Monica Centa, et al.

Noninvasive Neuromodulation of Peripheral Nerve Pathways Using Ultrasound and Its Current

Therapeutic Implications

Christopher Puleo and Victoria Cotero

Enteric Neuromodulation for the Gut and Beyond Yogi A. Patel and Pankaj J. Pasricha

Optogenetic Control of the Peripheral Nervous System

Rui B. Chang

Closed-Loop Neuromodulation in Physiological and Translational Research

Stavros Zanos

Electrical Impedance Methods in Neuromuscular Assessment: An Overview

Seward B. Rutkove and Benjamin Sanchez

Optogenetic Medicine: Synthetic Therapeutic

Solutions Precision-Guided by Light

Haifeng Ye and Martin Fussenegger

Technobiology's Enabler: The Magnetoelectric Nanoparticle

Sakhrat Khizroev
Bioelectronic Medicine: From Preclinical Studies on the Inflammatory Reflex to New Approaches in Disease Diagnosis and Treatment

Valentin A. Pavlov, Sangeeta S. Chavan and Kevin

J. Tracey

Vagus Nerve Stimulation and the Cardiovascular System

Michael J. Capilupi, Samantha M. Kerath and

Lance B. Becker

Harnessing the Inflammatory Reflex for the

Treatment of Inflammation-Mediated Diseases

Yaakov A. Levine, Michael Faltys and David Chernoff

Recording and Decoding of Vagal Neural Signals Related to Changes in Physiological Parameters and Biomarkers of Disease Theodoros P. Zanos

Restoring Movement in Paralysis with a

Bioelectronic Neural Bypass Approach: Current

State and Future Directions Chad E. Bouton

Bioelectronic Medicine--Ethical Concerns Samuel Packer, Nicholas Mercado and Anita Haridat

Use of Bioelectronics in the Gastrointestinal Tract Larry Miller, Aydin Farajidavar and Anil Vegesna

Vagus Nerve Stimulation at the Interface of BrainGut Interactions

Bruno Bonaz, Valérie Sinniger and Sonia Pellissier

For additional articles in this collection, see http://perspectivesinmedicine.cshlp.org/cgi/collection/ 